\title{
Construcción del significado psicológico del concepto ciencia por parte de grupos universitarios de Pedagogía
}

Construction of the psychological meaning of the concept of science by university groups of Pedagogy

\section{Volumen 18, Número 3 \\ Setiembre-Diciembre}

pp. $1-20$

Este número se publica el 1 de setiembre de 2018

DOI: https://doi.org/10.15517/aie.v18i3.33550

Cristián M. Aguilar Correa

Felipe J. Marín Isamit

Revista indizada en REDALYC, SCIELO

Revista distribuida en las bases de datos:

LATINDEX, DOAJ, REDIB, IRESIE, $\underline{\text { CLASE}}$ DIALNET, SHERPA/ROMEO, QUALIS-CAPES, MIAR

Revista registrada en los directorios:

ULRICH'S $, \underline{R E D I E}, \underline{\text { RINACE}}, \underline{\text { OEI }}, \underline{\text { MAESTROTECA }}, \underline{\text { PREAL, }}$ 


\title{
Construcción del significado psicológico del concepto ciencia por parte de grupos universitarios de Pedagogía
}

Construction of the psychological meaning of the concept of science by university groups of Pedagogy

\author{
Cristián M. Aguilar Correa ${ }^{1}$ \\ Felipe J. Marín Isamit ${ }^{2}$
}

\begin{abstract}
Resumen: El estudio tuvo por objetivo analizar y describir los significados psicológicos que posee un grupo de estudiantes universitarios de Pedagogía de Educación General Básica sobre el concepto ciencia. Ello resulta importante, pues los constructos que desarrollarán las futuras personas docentes caracterizarán los acontecimientos que ocurrirán en el aula. La muestra, intencionada no probabilística, estuvo compuesta por 169 estudiantes; de ellos 85 pertenecían a la carrera de Pedagogía en Educación General Básica y 84, a la carrera de Pedagogía en Educación General Básica, mención Lenguaje, Matemática, Ciencias Sociales y Ciencias Naturales de la Universidad Católica del Maule, Chile. La técnica de trabajo utilizada en esta investigación de tipo mixta, fueron las Redes Semánticas. Esta herramienta busca identificar la influencia de la cultura en la construcción psicológica de identidades. Los resultados revelan que el estudiantado posee significados comunes en torno a la ciencia. Se constata una fuerte tendencia a simbolizarla con ideas vinculadas hacia el conocimiento de naturaleza conceptual, la investigación desde la perspectiva del método científico y el hacer científico fundamentalmente circunscrito al trabajo llevado a cabo en el laboratorio tradicional de ciencias. Finalmente, significados vinculados hacia una ciencia más histórica, problematizadora y humanizadora están ausentes en los nodos conceptuales surgidos.
\end{abstract}

Palabras clave: psicología, ciencia, docentes, enseñanza superior.

\begin{abstract}
The objective of the study was to analyze and describe the psychological meanings of a group of university students of Pedagogy Elementary Education about the concept of science. This is important to understand because the constructs that future teachers will develop will characterize the events that will happen in the classroom. The sample, intentional non-probabilistic, was composed of 169 students; Of these, 85 belonged to the Pedagogy in Elementary Education, and 84 to the Pedagogy in Elementary Education, mention of Language, Mathematics, Social Sciences and Natural Sciences of the Universidad Católica del Maule, Chile. The work technique used in this mixed type research was the Natural Semantic Networks (Valdez, 2005). Tool that seeks to identify the influence of culture in the psychological construction of identities. The results reveal that the student has common meanings around science. There is a strong tendency to symbolize it with ideas linked to knowledge of a conceptual nature, research from the perspective of the scientific method and scientific research fundamentally circumscribed to the work carried out in the traditional science laboratory. Finally, meanings linked to a more historical, problematizing and humanizing science are absent in the conceptual nodes that have arisen.
\end{abstract}

Key words: psychology, science, teachers, higher education.

\footnotetext{
1 Académico del Departamento de Formación Inicial Escolar, Facultad de Ciencias de la Educación, Universidad Católica del Maule, Chile. Doctor en Didáctica de las Ciencias Experimentales, Universidad Complutense de Madrid. Dirección electrónica: caguilar@ucm.cl

${ }^{2}$ Académico de la Facultad de Ciencias de la Educación, Universidad Católica del Maule, Chile. Magíster en Educación de las Ciencias Mención Química, Universidad de Talca, Chile.Dirección electrónica: fmarin@ucm.cl
}

Articulo recibido: 20 de noviembre, 2017

Enviado a corrección: 6 de marzo, 2018

Aprobado: 21 de mayo, 2018 


\section{Introducción}

En la actualidad, se exponen numerosas investigaciones que buscan orientar la enseñanza de las ciencias en los diferentes niveles de la educación formal; existe, al mismo tiempo, un amplio consenso sobre la trascendencia que implica en la ciudadanía saber sobre ciencias. (González, Martínez y Martínez, 2009; Haberman, 2006; Hattie, 2003; Zahur, Barton y Upadhyay 2002). Las preguntas: ¿Cómo se construyen las ciencias?, ¿qué las hace particulares respecto de otras áreas del conocimiento? y ¿cómo influyen, o bien, son influenciadas por la cultura? (Lederman, 1992; Matthews, 2000; Sanmartí, 2009) son fundamentales en la comprensión epistémica y didáctica de esta importante área del saber.

Enseñar y aprender ciencias tiene que ver con actividades pedagógicas que posibilitan la apropiación de herramientas culturales y de nuevas formas de entender el mundo. (Henao, Silva, Moreira, 2011. p. 237). Hacer ciencias, por lo tanto, significa poder discutir, razonar, argumentar, criticar y justificar hipótesis. Sin embargo, los resultados de pruebas llevadas a cabo en Chile, tanto a nivel nacional como internacional, revelan que, en general, el estudiantado solo alcanza niveles de aprendizaje que van de lo muy simple y elemental a aprendizajes intermedios (Agencia de la Calidad de la Educación, 2013; 2014; 2015; UNESCO, 2013). Los escolares aplican estos saberes para explicar situaciones en contextos que les resultan muy sencillos, para plantear conclusiones simples y desarrollar interpretaciones del "mundo" convergentes y muy literales (Cariola, Cares, Lagos, Covacevich, Gubler, 2009). Posiblemente, las ideas más plausibles de esta problemática se fundamenten en acciones de enseñanza enfocadas a una educación científica planteada únicamente desde el paradigma tradicional (Pujalte, Bonan, Porro y Adúriz-Bravo, 2015) en donde el estudiantado actúa como mero receptor de una información fragmentada e inconexa (Ballenilla, 1992; Pomeroy, 1993; Porlán, 1989; Powell, 1994).

Respecto de la transformación de la enseñanza científica en los territorios escolares, existe evidencia de que el trabajo del cuerpo docente es determinante en el logro de estos objetivos, y la vía más efectiva para desarrollar una real alfabetización científica (González, et al. 2009; Haberman, 2006; Hattie, 2003; Zahur et al., 2002). No obstante, a pesar de los cambios producidos en la filosofía de la ciencia y en su didáctica, la enseñanza llevada a cabo por los maestros, sobre todo en niveles básicos y medios, continúa reflejando un trabajo cimentado desde significados psicológicos que determinan una didáctica transmisiva y una epistemología positivista (García-Carmona, Vázquez, y Manassero, 2011; Hodson, 1988; Pujalte et al., 2015). 
He aquí la importancia de esta investigación, el poder analizar y describir los significados psicológicos sobre ciencia que posee un grupo de estudiantes universitarios de Pedagogía de Educación General Básica. A partir de estas premisas, es posible inferir que la imagen de ciencia que posee cada docente en formación irisará sus futuras decisiones pedagógicas guiando y condicionando los acontecimientos didácticos que pudieran ocurrir en el aula (Aguilar, 2017; García et al., 2011).

\section{Referentes teóricos}

La profesora Beatriz Macedo, en su prólogo escrito para el informe del Segundo Estudio Regional Comparativo y Explicativo (SERCE) hacia énfasis en su reflexión, indica que:

La concepción de la enseñanza y del aprendizaje ha sufrido cambios significativos en los últimos años, con importantes consecuencias sobre la manera de entender cómo los estudiantes aprenden y, por lo tanto, sobre las posibles metodologías a desarrollar en las aulas. Estos cambios van de la mano con las nuevas concepciones de Ciencia y, por lo tanto, de educación científica. (UNESCO, 2010, p.12)

En Latinoamérica existe un amplio consenso sobre la importancia de la enseñanza de las ciencias naturales como un área del saber, que ayuda a los ciudadanos a mejorar su calidad de vida y les permite desenvolverse con éxito en un ambiente de constante cambio y trasformación (UNESCO, 2013).

La mayor parte de los países hace énfasis en el desarrollo de competencias científicas básicas como una manera de fomentar el pensamiento crítico, es decir, desarrollar la capacidad de ser objetivos, de encontrar inconsistencias en los argumentos y de valorar la información presentada ante la toma de decisiones. (UNESCO, 2015, p. 78)

En esta misma línea, podemos indicar que la calidad en la Formación Inicial Docente (FID) ha sido un área de creciente interés, tanto para los gobiernos como para las instituciones responsables de su formación.

Lo anterior cobra relevancia si se considera que la FID explica o debería explicar la calidad del quehacer de la persona docente puesta a prueba, ya sea, en los procesos de práctica como en los contextos de inserción profesional (Fuentealba, 2003; Paquay, Altet, Charlier y Perrenoud, 2010). 
Para su aprendizaje, la futura persona docente se despliega en dos niveles de realidad: uno que tiene relación con su condición de docente en el aula, y una segunda dimensión en donde reflexiona sobre esa práctica áulica. (Galaz, Fuentealba, Cornejo y Padilla, 2011). Es precisamente en estos escenarios donde se enfatiza la importancia y trascendencia que tienen los buenos maestros sobre la calidad y pertinencia de los aprendizajes de sus estudiantes (Ministerio de Educación, 2005).

En Chile, lo que ha permitido dar pasos importantes en materia de FID ha sido la elaboración y puesta en marcha de Estándares Orientadores (disciplinarios y pedagógicos) para las carreras de pedagogía a partir del año 2011, inicialmente con énfasis en los egresados de Pedagogía en Educación General Básica, para, desde aquí, transitar hacia otras pedagogías. Los estándares tienen por misión servir de guías a las carreras educativas estableciendo las capacidades que los egresados deben demostrar al enseñar. Es así que los estándares representan un qué, referido a un conjunto de aspectos que se deberían observar en el desempeño de un futuro profesor o profesora, y también establecen un cuánto, lo que permite poder evaluar qué tan lejos o qué tan cerca se encuentra un nuevo docente de alcanzar un determinado desempeño (Ministerio de Educación, 2011).

En el caso de la enseñanza científica, los estándares relacionados con las competencias que deben movilizar los futuros académicos sobre los estudiantes de educación básica, instruyen sobre las variables que afectan el aprendizaje en ciencias, como así también, plantean las capacidades que debiera demostrar el profesorado para enseñar esta trascendental área del conocimiento. De igual modo, orientan al futuro docente para que pueda promover competencias científicas y estimule a los estudiantes a que puedan establecer relaciones entre la ciencia y su vida cotidiana (Ministerio de Educación 2011).

El Ministerio de Educación (2011), en el estándar 2, propone que:

El futuro profesor o profesora, sabe que la actividad científica pretende explicar el mundo natural y material, y que el conocimiento desarrollado permite hacer predicciones sobre los fenómenos naturales e intervenir en ellos. Comprende y utiliza las ideas fundamentales de las Ciencias Naturales, identifica los aportes de las disciplinas centrales que la componen (Física, Química y Biología) y comprende las relaciones que se dan entre ellas para explicar o interpretar los fenómenos naturales en su complejidad. Finalmente, comprende la naturaleza social de la producción del conocimiento científico y la influencia recíproca entre el desarrollo científico, tecnológico y social. (p.142) 
Esto se ve complementado con el estándar 9, el cual establece que:

El futuro profesor o profesora, sabe promover el desarrollo de actitudes y habilidades propias del pensamiento y quehacer científico, en concordancia con los contenidos y objetivos del currículo y con los intereses de los estudiantes. Puede diseñar actividades donde los estudiantes elaboren preguntas, hipótesis e interpretaciones.

Está consciente de la importancia del trabajo colaborativo entre los estudiantes y diseña instancias para ello. Sabe cómo estimular a los estudiantes a complementar sus observaciones con información científica proveniente de distintas fuentes y así fundamentar sus conclusiones. Finalmente, es capaz de presentar a los estudiantes el conocimiento científico como explicaciones o interpretaciones de un fenómeno y no como una verdad inalterable. Diseña actividades de aprendizaje e instrumentos para identificar los logros alcanzados por los alumnos en el desarrollo de dichas habilidades. (Ministerio de Educación, 2011, p.156)

Otra política chilena orientadora en la FID tiene relación con las Bases Curriculares para la enseñanza básica del año 2012, en ellas se establece que "el conocimiento científico es por definición, dinámico, mutable y transitorio, pues siempre está la posibilidad de que, en el futuro, nuevas explicaciones refuten a las que hoy damos por ciertas" (Ministerio de Educación. Bases Curriculares Educación Básica, 2012, p. 138). Al respecto, podemos señalar que estas orientaciones en la educación básica:

Están enfocadas a la alfabetización científica de todos los alumnos. Esto corresponde a la capacidad de los estudiantes para aplicar en su vida ordinaria los conocimientos y las habilidades aprendidas, hacerse preguntas sobre distintos fenómenos y obtener conclusiones basadas en la evidencia. Lo anterior les permitirá comprender el mundo natural, tomar decisiones informadas dentro de él y llevar dichas decisiones a diversas actividades humanas que afecten a su familia y comunidad. (Ministerio de Educación, 2012, p. 139)

Ahora bien, es importante advertir que el paso de un conocimiento cotidiano a un conocimiento formal no solo requiere de una reestructuración de los niveles pedagógicos y didácticos, sino también de una reestructuración del armazón epistemológico desde donde son formados los potenciales docentes. Por tanto, cabe hacerse la pregunta ¿cómo 
construimos significados los individuos?. Valdés (2005) sostiene que el significado psicológico corresponde a una unidad fundamental de organización cognoscitiva, cargada de elementos afectivos y de conocimientos, los cuales reflejan, de una u otra forma, la imagen de universo que construimos las personas.

El significado psicológico constituye un código de información relativo a un objeto en particular, pero, que también depende de la base social que se tenga (Valdez, 2005). Dentro de este terreno se pueden encontrar inmersas las normas morales, las creencias, los conceptos, los mitos, etc. En efecto, toda sociedad puede ser estudiada a través de la relación que se establece entre sus miembros y los significados psicológicos prevalecientes en la cultura de dichos sujetos (Valdez, 2005).

Vygotski (2009) reconoce la existencia de un mundo interno que los sujetos edificamos a partir de un proceso de internalización de un mundo externo. Esta construcción se halla en las distintas conexiones que el ser humano genera a partir de los signos, los cuales cumplen un papel primordial en la comprensión y construcción de los significados. Vygotski sostiene que si los signos se encuentran en la cultura, los significados son parte consustancial de ella. En efecto, los significados se construyen a través de la internalización de los procesos psicológicos superiores, cuyo valor radica en la capacidad de crear estímulos artificiales que pasan finalmente a ser causas inmediatas de nuestras conductas (Vygotski, 2009). Toda realidad es representada individual o colectivamente y es reconstruida e integrada en un sistema de valores dependiente de una historia particular y de un contexto social específico (Abric, 2004).

Toda significación es así, una forma de visión global y unitaria de un objeto, pero también de un sujeto. Esta significación reestructura la realidad para, a la vez, permitir una integración de las características objetivas del objeto, de las experiencias anteriores del sujeto y de su sistema de normas y actitudes. Esto permite definir la significación del objeto como una visión funcional del mundo, la cual permite al individuo o al grupo conferir sentido a sus conductas y entender la realidad mediante su propio sistema de referencias, y adaptar y definir, de este modo, un lugar para sí (Abric, 2004).

Por tanto, los estudiantes de pedagogía no son páginas en blanco sobre los que hay que imprimir un saber, ellos poseen su propias concepciones y significados del mundo en el que viven y transitan (Pozo y Gómez, 2001). Precisamente la evolución progresiva de esas representaciones es lo que va constituyendo distintos niveles de conocimiento, pero siempre desde una perspectiva individual, reconocida y cimentada fuertemente en un contexto 
sociocultural. En consecuencia, el proceso educativo, en la FID, debe atender todos los objetivos que contribuyan al completo desarrollo del futuro docente, incluidas sus dimensiones: pedagógica, disciplinar, social, afectiva, artística, cívica, emocional, imaginativa, crítica, ambiental, etc. (Martín, 1992).

Finalmente, es importante señalar que toda realidad es representada, ya sea por un individuo o un colectivo social y reconstruida en un sistema cognitivo integrado a un sistema de significados fuertemente influidos por una historia individual cargada de elementos figurativos (Abric, 2004).

\section{Marco metodológico}

\subsection{Muestra}

Se consideró una muestra intencionada compuesta por 169 estudiantes. 85 pertenecían a la carrera de Pedagogía en Educación General Básica y 84 pertenecían a la carrera de Pedagogía en Educación General Básica, mención Lenguaje, Matemática, Ciencias Sociales y Ciencias Naturales de la Universidad Católica del Maule-Chile (Ver Tabla 1).

Tabla 1

Número de estudiantes por carrera, según sexo, Universidad Católica del Maule-Chile, AÑO 2017

\begin{tabular}{lcccc}
\hline \multicolumn{1}{c}{ Carrera } & \multicolumn{2}{c}{ Estudiantes } & Total \\
\cline { 3 - 4 } & Hombres & Mujeres & \\
\hline $\begin{array}{l}\text { Ped. en Educación General Básica (mención: } \\
\begin{array}{l}\text { Matemática, Lenguaje y Comunicación, } \\
\text { Historia y Geografía y Ciencias Naturales) }\end{array}\end{array}$ & 6 & 78 & \multirow{2}{*}{84} \\
\hline Ped. en Educación General Básica & 8 & 77 & 85 \\
\hline & Total & 14 & 55 & 169 \\
\hline & Porcentaje & $9 \%$ & $91 \%$ & $100 \%$ \\
\hline
\end{tabular}

Fuente: Elaboración propia de los autores (2017).

\subsection{Tipo de investigación}

La investigación se enmarca dentro de un enfoque mixto fundamentado a partir de un diseño no experimental de tipo transeccional descriptivo. 


\subsection{Instrumento}

El instrumento utilizado para la recolección de la información fue la técnica de Redes Semánticas Naturales (RSN), (Valdez, 2005), del tipo evocación y jerarquización (Abric, 2004). Esta técnica, por un lado, intenta dar una explicación acerca de cómo ocurre la construcción de los significados en los individuos persiguiendo poder acceder tanto al contenido como a la estructura de la representación que significan los sujetos (Mazzitelli, Guirado y Chacoma, 2011). Y, por otro lado, poder identificar la visión del mundo que los individuos construyen y que utilizan para actuar o tomar posición en la dinámica de las interacciones sociales y las determinantes de las prácticas sociales (Abric, 2004).

Para las RSN, la información almacenada en la memoria está organizada en nodos conceptuales que, en conjunto, producen significados de organización cognoscitiva, cargados de elementos figurativos. Estos crean un código personal de reacción, con lo cual reflejan la imagen que los sujetos nos hacemos del mundo que transitamos. Las RSN brindan una metodología práctica de acción que nos permite poder vislumbrar cómo los sujetos organizan el conocimiento en su estructura cognitiva (Ausubel, 2009) y cómo, a partir de esta internalización (Vygotski, 2009), el significado es construido desde un escenario cargado de entidades psicológicas individuales matizadas por un poderoso medio sociocultural.

Respecto de cómo abordar el instrumento, la idea es que los participantes puedan definir con la mayor precisión posible un concepto nodal dado (Ciencia). Para ello, deben utilizar un mínimo de cinco palabras que posteriormente deben jerarquizar de acuerdo con la importancia que ellos consideren que tiene su red conceptual en relación con el estímulo propuesto. De esta manera, asignan el número 1 a la palabra más cercana, el 2 a la que sigue y así repetidamente hasta terminar con el número 5 .

\section{Análisis de los datos}

Para una mayor claridad en la presentación de los resultados se utilizará la propuesta de RSN indicada por Lagunes, (1993) y Valdez (2005).

Cabe mencionar, que el cómputo y el procesamiento cuantitativo de la información se fundamentan a partir de una estadística descriptiva general. Particularmente, los indicadores puntualizados por los autores son: Conjunto SAM, grupo de las diez palabras definidas por los estudiantes que obtienen los mayores valores M; valor J o Tamaño de la Red (TR), valor que se obtiene sumando el número de palabras que fueron definidas por cada uno de los 
grupos respecto del reactivo dado.

Peso Semántico (valor M), corresponde al valor de la frecuencia, asignado por los estudiantes a cada concepto, multiplicado por el valor semántico dado por los autores de la técnica (Valor $5=1$; valor $4=2$; valor $3=3$; valor $2=4$ y valor $1=5$ ). Posteriormente, se presenta la Diferencia Semántica Cuantitativa (DSC), indicador (en porcentaje) de la distancia semántica que hay entre las diferentes palabras que conforman el núcleo de la red, este valor se obtiene mediante una proporción directa, que toma como inicio la palabra con el valor M mayor, la cual representa el 100\%.

Por su parte, los nodos de datos generados en las redes fueron codificados en un sistema mayor de categorías a objeto de visibilizar la variabilidad de los significados aparecidos y su correspondencia con familias conceptuales ligadas a la naturaleza de la ciencia (Fernández, Gil, Carrascosa, Cachapuz y Praia, 2002).

Finalmente, y con el objeto de obtener una nube general de conceptos, desde el cómputo de las frecuencias, se trabajó procesando la información a partir del programa en línea denominado "Nube de Palabras"4.

\section{Resultados}

Para el estudiantado de la carrera de Pedagogía en Educación General Básica, el núcleo de la red para el concepto Ciencia (ver Tabla 2) quedó dado por la palabra troncal: Investigación. Valor M de 199 y diferencia semántica cuantitativa (DSC) del 100\%. Seguidamente, y con distancias semánticas más o menos regulares, emergen los conceptos: Estudio y Experimentos. Valores M de 159 y 153 y DSC de $80 \%$ y $77 \%$, respectivamente. Luego y con valores M de 102 y DSC del 51\% figura el concepto Método. Seguidamente, surge una red de conceptos con distancias semánticas similares, estas palabras serían: Observación, Conocimientos y Descubrir. Con valores M entre 65 y 79 y DSC en los rangos $39 \%$ y $51 \%$. Por último, emergen los nodos conceptuales Naturaleza, Vida y Tecnología, con valores $\mathrm{M}$ cercanos a 35 y DSC entre los valores $17 \%$ y $23 \%$.

Cabe señalar que el tamaño de la red semántica (valor J) estuvo compuesto por un total de 63 definidoras.

\footnotetext{
${ }^{4}$ Disponible en https://www.nubedepalabras.es/
} 
Tabla 2

Peso Semántico y Diferencia Semántica Cuantitativa de los estudiantes, Universidad Católica del Maule-Chile, AÑO 2017

\begin{tabular}{lcc}
\hline \multicolumn{1}{c}{ Palabras definidoras } & Peso semántico & $\begin{array}{c}\text { Diferencia semántica } \\
\text { cuantitativa (\%) }\end{array}$ \\
\hline Investigación & 199 & 100 \\
\hline Estudio & 159 & 79 \\
\hline Experimentos & 153 & 76 \\
\hline Método & 102 & 51 \\
\hline Observación & 79 & 39 \\
\hline Conocimientos & 78 & 39 \\
\hline Descubrir & 65 & 32 \\
\hline Naturaleza & 45 & 22 \\
\hline Vida & 35 & 17 \\
\hline Tecnología & 33 & 17
\end{tabular}

Tamaño de la red $=63$

Fuente: Elaboración propia de los autores (2017).

Con respecto a la red semántica surgida desde los significados de los estudiantes de la carrera de Pedagogía en Educación General Básica con mención (ver Tabla 3), podemos indicar que el núcleo de la red quedó conformado por las palabras, Estudio e Investigación. Valores M de 243 - 223 y DSC de 100\% y 92\% respectivamente. Luego, surge el concepto Experimentos (valor M de 138 y DSC del 57\%). Seguidamente, emerge el concepto Conocimiento, con valores M de 83 y DSC del 34\%. Posteriormente, surge un grupo de tres palabras, con valores $M$ cercanos, estas palabras fueron: Descubrir, Metodología y Naturaleza. Con valores $M$ en los rangos $42-55$ y DSC, entre los valores $17 \%$ al $23 \%$.

Finalmente, aparecen los conceptos: Hipótesis, Exacta y Verdad con valores M en los rangos $28-33$ y DSC en los rangos $12 \%$ y $14 \%$. El tamaño de la red (valor J) estuvo compuesta por un total de 52 definidoras. 
Tabla 3

Peso Semántico y Diferencia Semántica Cuantitativa de los estudiantes, Universidad Católica del Maule-Chile, AÑO 2017

\begin{tabular}{lcc}
\hline \multicolumn{1}{c}{ Palabras definidoras } & Peso semántico & $\begin{array}{c}\text { Diferencia Semántica } \\
\text { Cuantitativa (\%) }\end{array}$ \\
\hline Estudio & 243 & 100 \\
\hline investigación & 223 & 92 \\
\hline Experimentos & 138 & 57 \\
\hline Conocimientos & 83 & 34 \\
\hline Descubrir & 55 & 23 \\
\hline Metodología & 55 & 23 \\
\hline Naturaleza & 42 & 17 \\
\hline Hipótesis & 33 & 14 \\
\hline Exacta & 31 & 13 \\
\hline Verdad & 28 & 12 \\
\hline Tamaño de la red $=\mathbf{5 2}$ & \\
\hline Fuente: Elaboración propia de los autores (2017).
\end{tabular}

A continuación, se presentan las redes conceptuales y sus correspondencias con las categorías (Fernández, et al. 2002).

Tabla 4

Categorías y redes conceptuales ligadas al significado de Ciencia

\begin{tabular}{llc}
\hline Categorías & Redes conceptuales asociadas & Porcentaje \\
& & 67 \\
\hline Ciencia rígida. & Experimentos & 23 \\
& Metodología & 13 \\
& Exacta & 12 \\
& Verdad & 39 \\
Ciencia aproblemática y ahistórica. & Observación & 14 \\
\cline { 2 - 3 } Ciencia exclusivamente analítica. & Hipótesis & 90 \\
& Estudio & 39 \\
\hline Ciencia con conocimientos & Descubrir & 96 \\
puramente acumulativos. & Investigación & 37 \\
& Conocimientos & 17 \\
\hline Ciencia histórica, contextual. & Tecnología & 17 \\
\hline & Naturaleza & 17
\end{tabular}

Fuente: Elaboración propia de los autores (2017), con información de Fernández et al. (2002).

Con respecto a los resultados obtenidos por medio de la nube de conceptos (ver Figura 1), podemos indicar que los nodos estudio e investigación concentran la mayor ponderación en términos de frecuencias acumuladas. 
Otro grupo de palabras surgido, revela los términos: experimentos, método, y conocimiento. Resulta interesante el surgimiento del nodo dado por los conceptos: descubrir y naturaleza. Como últimos vocablos, emergen las palabras: Observación, tecnología, vida, exacta, verdad e hipótesis. Lo anterior, y a la luz de los datos revelados, nos hace suponer que para estos futuros profesionales, la ciencia esta significada desde fundamentos epistémicos enraizados en el positivismo lógico. Al respecto, la investigación nos da a entender que en estas áreas, estudiantes y docentes muchas veces no han adquirido el nivel de comprensión deseado acerca de la construcción del conocimiento científico (Fernández et al., 2002).

Figura 1

Nube general de conceptos

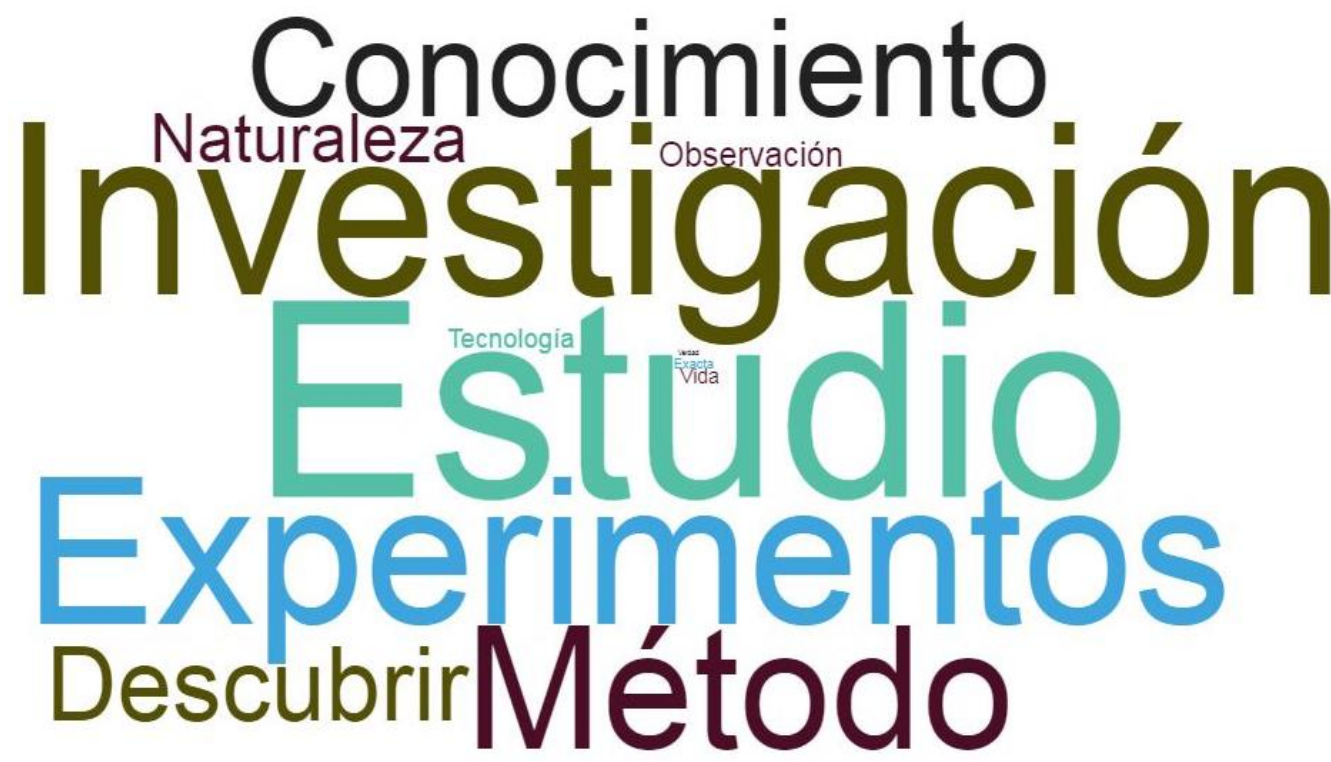

Fuente: Elaboración propia de los autores (2017).

Para los futuros profesores de Educación General Básica, la ciencia significa mayoritariamente Estudio e Investigación (ver Figura 1). Ambos conceptos sugieren la relevancia que simbolizan, para estos futuros profesionales, unas ideas de ciencia referidas a una concepción desde la tradicional concepción heredada de esta y lo complejo que resulta derribar estas concepciones desde la FID universitaria. Estos hallazgos coinciden con los encontrados por Flores, Caballero y Moreira (2013), al revelar que futuros docentes piensan que la investigación científica se inicia con la observación directa, y que esta es un 
proceso sin relación con las ideas apriorísticas del sujeto. Lo anterior, deja entrever que, para los futuros docentes es difícil advertir la función condicionante de las teorías y los saberes previos en la producción del conocimiento.

Por su parte, en un trabajo llevado a cabo por Vildósola, García y Catelló (2005), sobre la formación inicial del profesorado de ciencias, los autores encontraron una visión de ciencia expresada a Estudio. Quizás esta percepción de las personas docentes se funda en una concepción ingenua de la ciencia, mirada inminentemente atomizada/disciplinar y posiblemente derivada de los modelos empírico-tecnológicos que por tanto tiempo han sustentado la idea de que el método científico y la comprobación empírica son las únicas vías para la obtención de conocimiento científico.

Otros elementos interesantes son los nodos conceptuales Experimentos y Método (ver Figura 1). Al respecto, Fernández et al. (2002) afirman que esta imagen empirista de los docentes sobre la ciencia, que transmiten cuando enseñan, no es muy distinta de las imágenes socialmente admitidas. Este significado de ciencia persiste tanto en maestros como en estudiantes, permeando de forma negativa la enseñanza científica escolar. En efecto, se olvida el carácter tentativo de la ciencia, se ignora el papel primordial de las hipótesis, así como las crisis y las reformulaciones profundas del conocimiento científico en un contexto histórico "muy humano" y provisional. En este mismo sentido, Fernández et al (2002) señalan que los futuros maestros creen en el carácter experimental de la ciencia. Sin embargo, la relación del experimento con la teoría es vista de manera diferencial, simplemente se le atribuye una función comprobatoria de las hipótesis declaradas (Flores et al., 2013).

Respecto del Método (científico), los maestros lo conciben como un hacer "ritualizado" y "protocolar", consideran que su aplicabilidad desarropada de toda teoría asegurará resultados infalibles y verdaderos. Estas ideas contrastan con lo hallado por Flores et al. (2013) al trabajar con profesores en formación de biología y química. Los grupos estudiados conciben la existencia de un método científico como una secuencia lineal de pasos que hay que seguir. Los estudiantes consideran que este es el hacer que caracteriza a la ciencia y es el acto que le confiere a ella su definición de rigurosidad y objetividad (Vildósola et al., 2005). En este sentido, Toulmin (2003) plantea que no existe un método universal, autovalidante y obligatorio y que, inclusive, el mismo término método se ha tergiversado de su significado único que implica la persecución de un objetivo sin referirse propiamente a procedimientos específicos u obligatorios a seguir para alcanzarlo (p.133). 
Otro nodo interesante, surgido en las redes, ha sido el concepto Conocimientos (ver Figura 1). Este significado, tal vez, representa la idea más clásica que poseemos los sujetos sobre la ciencia (Flores, et al., 2013). Es importante señalar que hay dos estados en el conocimiento científico que deben ser diferenciados, uno el conocimiento consolidado, cuya mutabilidad no ocurre en periodos cortos, y el conocimiento de frontera, que cambia y muta constantemente por estar en el centro de la investigación científica del momento (Flores et al., 2013). Es preciso señalar que el estudio arroja significados que representan ideas más encaminadas a una ciencia inmutable y atemporal que a una ciencia transitoria y provisional.

Otros elementos interesantes son los nodos conceptuales Observación y Descubrir. En este sentido, podemos indicar que a partir de la información reportada creemos que los docentes tienen una concepción de ciencia basada en la búsqueda de certezas, en la trasmisión de un conocimiento acabado y en donde la observación y la experimentación científica se limitarían a comprobar una verdad expuesta (Sanmartí, 2009). De acuerdo con Pujalte et al (2015) preexiste una buena carga teórica acerca del alcance de la observación y el rol que juegan los aspectos subjetivos, valóricos y contextuales en la producción del conocimiento científico. No obstante, la visión de ciencia presente en el estudiantado es de carácter positivista, algorítmica e infalible (Fernández, et al., 2002).

Otro grupo conceptual interesante, surgido en las redes semánticas, nos habla de palabras que relevan la ciencia a conceptos como Exacta y Verdadera. Esta mirada de ciencia, un tanto tradicional, contrasta con lo que proponen Porlán y Martín del Pozo (2004). Ellos llamarían a este tipo de ciencia, ciencia del saber y del conocer. En efecto, ciencia generada a partir de procesos instrumentalistas, cuya operacionalización se circunscribe a una metodología de trabajo en donde los estudiantes, hacen ciencias, aplicando rigurosa y sistemáticamente los pasos que establece el método científico. En esta línea, Fourez (2008) advierte que esta idea de ciencia simboliza una visión del mundo siempre unívoca y estandarizada.

Por último, las personas docentes en formación representan la ciencia desde elementos figurativos ligados a la Naturaleza. Posiblemente los estudiantes consideran la ciencia como una adición de disciplinas que tienen por misión explicar los fenómenos de la naturaleza. A lo mejor, esta visión ingenua considera la ciencia como una colección de conocimientos organizados, en este caso, principalmente sobre la naturaleza y el universo (Fernández et al., 2002). Lo anterior, hace suponer que las personas docentes en formación ignoran las complejas relaciones suscitadas en la ciencia (Fernández, Gil, Valdés, Vilches, 
2005) y olvidan, quizás, otros elementos imbricados en el quehacer científico, como podrían ser algunos elementos tecnológicos, sociales, culturales e históricos (Toro-Baquero, 2014).

\section{Conclusiones}

Hay elementos en común en los significados psicológicos que las personas docentes en formación tienen respecto de la epistemología y la didáctica de las ciencias. Los significados como: experimentos, conocimientos, estudio, método, entre otros son recurrentes en ambas redes conceptuales. Del mismo modo, significados vinculados hacia una ciencia más histórica, problemática-problematizadora, humana y humanizadora quedan relegados a un segundo plano, o bien están ausentes de dichos nodos conceptuales. Existe una tendencia a significarla con ideas vinculadas a la investigación, al estudio, el conocimiento y el empirismo. Las evidencias del estudio contrastan la fuerte disposición que hay al comprender la enseñanza científica escolar desde el trabajo experimental reducido, quizás, al mero ejercicio algorítmico en el laboratorio tradicional.

Igualmente, hay una predisposición a representar la ciencia con el conocer y el saber. En este sentido, estamos frente a significados psicológicos fuertemente anclados al paradigma educativo tradicional, modelo que caracteriza el hacer educativo desde un escenario pedagógico permeado por la predicción, el control y la regulación, que centra el hacer en la figura del maestro y relega a segundo plano el protagonismo de los estudiantes. Elementos fuertemente criticados hoy desde el nuevo planteamiento epistemológicodidáctico del quehacer científico en la escuela primaria.

Existe, por otro lado, una fuerte tendencia, en los futuros docentes de Educación General Básica, a figurar la ciencia desde el método científico. Hacer ciencias es aplicar rigurosamente esta metodología. Este acto, posiblemente, asegura resultados válidos, lógicos y claros. Este hacer, casi "aislado" de la enseñanza y de todo significado cultural, conlleva, a la larga, a que los futuros docentes, cuando enseñen, transmitan una imagen de ciencia a-histórica y a-humana, con lo cual materializan un quehacer científico desinteresado y desarraigado de los fenómenos socioculturales.

Podemos indicar que los y las docentes en formación simbolizan la ciencia desde elementos representativos unidos a la idea de naturaleza. La ciencia es naturaleza, olvidando, quizás, otros elementos imbricados en el tejido del quehacer científico, como podrían ser algunos elementos sociales, culturales e históricos cuya acción reside en estar permeando constantemente la epistemología y la didáctica de las ciencias. Asimismo, 
podemos indicar que, dentro de las redes semánticas surgidas, no figuraron elementos epistemológicos y didácticos vinculados a nodos conceptuales, tales como error, provisionalidad, refutación, incertidumbre, etc., elementos figurativos clave que caracterizan la nueva epistemología de la ciencia.

Resulta importante señalar que el mayor flujo de información correspondió a datos declarados por futuras profesoras (155) lo que representa un $91 \%$ de la muestra total. Ello nos desafía y nos debe movilizar a cambiar el foco epistemológico y didáctico de la construcción de conocimiento científico desde las mujeres, y al mismo tiempo desarroparnos de esa mirada de la ciencia desde la concepción heredada para dar pasos a una enseñanza científica escolar más histórica, contextual y por sobre todo, humana y humanizadora.

Bajo este panorama de resultados es fundamental llevar a cabo al interior de la universidad, una profunda reflexión y un amplio debate referido a los elementos psicosociales que condicionan y determinan la construcción del conocimiento científico y su naturaleza didáctica en la FID.

Atender sus resultados en los ámbitos formativos de los futuros maestros y maestras es hacernos cargo de mejores procesos de enseñanza y aprendizaje vinculados a la ciencia escolar, sobre todo, los referidos a la formación de la población estudiantil de los niveles educativos primarios.

El desafío que queda planteado es ampliar el estudio hacia los docentes de ciencias en ejercicio con el fin de poder conocer sus significados y comprender cómo estos son transferidos al aula. Sería, al mismo tiempo, una interesante experiencia poder trabajar tanto con docentes que laboran en territorios escolares urbanos como aquellos que lo hacen en comunidades educativas en contextos de ruralidad.

\section{Referencias}

Abric, Claude. (2004). Prácticas sociales y representaciones. México: Coyoacán.

Agencia de la Calidad de la Educación. (2011). Resultados TIMSS 2011 Chile. Estudio Internacional de Tendencias en Matemática y Ciencias. Santiago de Chile: División de estudios. Recuperado de https://s3.amazonaws.com/archivos.agenciaeducacion.cl/documentosweb/Estudios+Internacionales/TIMSS/Informe+Nacional+de+Resultados+TIMSS+2011. pdf 
Agencia de la Calidad de la Educación. (2014). Informe técnico SIMCE 2014. Santiago de Chile: División de estudios. Recuperado de http://archivos.agenciaeducacion.cl/InformeTecnicoSimce 2014.pdf

Agencia de la Calidad de la Educación. (2015). PISA 2015. Programa para la evaluación de internacional de los estudiantes OCDE. Santiago de Chile. Recuperado de http://archivos.agenciaeducacion.cl/Resultados PISA2015.pdf

Aguilar, Cristián. (2017). La construcción del significado psicológico del concepto profesor o profesora por parte de grupos universitarios de estudiantes de pedagogía. Revista Electrónica Educare, 21(1). Doi: http://dx.doi.org/10.15359/ree.21-1.13

Ausubel, David. (2009). Adquisición y retención del conocimiento. Una perspectiva cognitiva. Barcelona: Paidós.

Ballenilla, Fernando. (1992). El cambio de modelo didáctico, un proceso complejo. Investigación en la Escuela, (18), 43-68.

Cariola, Leonor, Cares, Gabriela, Lagos, Ema, Covacevich, Catalina y Gubler, Johanna. (2009). ¿Qué nos dice PISA sobre la educación de los jóvenes en Chile? Nuevos análisis y perspectivas sobre los resultados en PISA 2006. Santiago de Chile: Ministerio de Educación.

Fernández Isabel, Gil, Daniel, Carrascosa, Jaime, Cachapuz, Antonio y Praia, Joao. (2002). Visiones deformadas de la ciencia transmitidas por la enseñanza. Enseñanza de las Ciencias, 20(3), 477-488.

Fernández, Isabel, Gil, Daniel, Valdés, Pablo y Vilches, Amparo. (2005). ¿Qué visiones de la ciencia y la actividad científica tenemos y transmitimos? La superación de las visiones deformadas de la ciencia y la tecnología: Un requisito esencial para la renovación de la educación científica. Santiago: Oficina Regional de Educación para América Latina y el Caribe, OREALC/UNESCO.

Flores, Julia, Caballero, María y Moreira, Marco. (2013). Ideas epistemológicas sobre la naturaleza de la ciencia de docentes en formación de Biologia y de química. Revista curriculum, (26), 101-133.

Fourez, Gerard. (2008). Cómo se elabora el conocimiento, la epistemología desde un enfoque socioconstructivista. Madrid: Narcea.

Fuentealba, Rodrigo. (2003). La Inserción Profesional de Profesores Principiantes de Enseñanza Media de la Región Metropolitana (Tesis de Doctorado). Pontificia Universidad Católica de Chile, Santiago, Chile.

Galaz, Alberto, Fuentealba, Rodrigo, Cornejo, José y Padilla, Alfonso. (2011). Estrategias reflexivas. La formación de profesores y de formadores de profesores. Santiago: Lom.

García-Carmona, Antonio, Vázquez, Ángel y Manassero, María. (2011). Estado actual y perspectivas de la enseñanza de la naturaleza de la ciencia: una revisión de las creencias y obstáculos del profesorado. Enseñanza de las Ciencias, 29(3), 403- 412. 
González, Corina, Martínez, María y Martínez, Carolina. (2009). La Educación Científica como apoyo a la movilidad social: desafíos en torno al rol del profesor secundario en la implementación de la indagación científica como enfoque pedagógico. Estudios Pedagógicos, 35(1), 63-78. Doi: http://dx.doi.org/10.4067/S0718-07052009000100004

Haberman, Martin. (2006). The special role of science teaching in schools serving diverse children in urban poverty. En: Flick, L \& N. Lederman (eds.), Scientific inquiry and the nature of science: Implications for teaching, learning, and teacher education (pp. 3753). Netherlands: Springer.

Hattie, John. (2003). Teachers make a difference: What is the research evidence? Paper presented at the Australian Council for Educational Research Annual Conference on Building Teacher Quality, Melbourne.

Henao, Berta, Silva, María y Moreira, Marco. (2011). La educación en ciencias desde la perspectiva epistemológica de Stephen Toulmin. Latin-American Journal of Physics Education, 5(1). Recuperado de http://www.lajpe.org/index march11.html

Hodson, Derek. (1988). Toward a philosophically more valid science curriculum. Science Education, 72(1), 19-40.

Lagunes, Isabel. (1993). Las redes semánticas naturales, su conceptualización y su utilización en la construcción de instrumentos. Revista de Psicología Social y Personalidad, 9(1), 81-97.

Lederman, Norman. (1992). Students' and teachers' conceptions of the nature of science: A review of the research. Journal of Research in Science Teaching, 29(4), 331-359.

Martín, Francisca. (1992). Curso interdisciplinar de educación ambiental. Madrid: Editorial Complutense.

Mazzitelli, Claudia, Guirado Ana y Chacoma, Mónica. (2011). La docencia y la enseñanza de las ciencias: análisis de las representaciones de profesores. Revista de Orientación Educacional, 25(48), 77-94.

Matthews, Michael. (2000). Time for science education: How teaching the history and philosophy of pendulum motion can contribute to science literacy. Nueva York: Plenum Publishers.

Ministerio de Educación Chile. (2011). Estándares Orientadores para Egresados de Carreras de Pedagogía en Educación Básica. Centro de Perfeccionamiento, Experimentación e Investigaciones Pedagógicas. Ministerio de Educación. Chile.

Ministerio de Educación Chile. (2012). Bases Curriculares Educacion Básica. Santiago de Chile.

Ministerio de Educación Chile. (2005). Bases curriculares de la educación parvularia. Santiago de Chile: El Ministerio, Unidad de curriculum y evaluación. 
Paquay, Leopold, Altet, Marguerite, Charlier, Evelyne y Perrenoud, Philippe. (2010). La formación profesional del maestro. Estrategias y competencias. México D.F.: FCE.

Pomeroy, Deborah. (1993). Implications of teachers' beliefs about the nature of science: Comparison of the beliefs of scientist, secondary science teachers, and elementary teachers. Science Education, 77(3), 261-278.

Porlán, Rafael. (1989). Teoría del conocimiento, teoría de la enseñanza y desarrollo profesional. Las concepciones epistemológicas de los profesores (Tesis Doctoral inédita). Universidad de Sevilla, Sevilla, España.

Porlán, Rafael y Martín Del Pozo, Rosa. (2004). The conceptions of in-service and prospective primary school teachers about the teaching and learning of science. Journal of Science Teacher Education, 15(1), 39-62.

Powel, Richard. (1994). From field science to classroom science: A case study constrained emergence in a second-career science teacher. Journal of Research in Science Teaching, 31(3), 273- 291.

Pozo, Juan y Gómez, Miguel. (2001). Aprender y enseñar ciencias (3ª ed.). Madrid: Morata.

Pujalte, Alejandro, Bonan, Leonor, Porro, Silvia y Adúriz-Bravo, Agustín. (2015). Las imágenes inadecuadas de ciencia y de científico como foco de la naturaleza de la ciencia: estado del arte y cuestiones pendientes. Ciência \& Educação, 20(3), 535-548.

Sanmartí, Neus. (2009). Didáctica de las ciencias en la educación secundaria obligatoria. Madrid: Síntesis.

Toro-Vaquero, Javier. (2014). ¿Qué visiones de CTS tienen los docentes de 5ํ y 9o grado de Colombia? Y ¿cuál es su relación con los estándares de ciencias del Ministerio de Educación Nacional? Ciência \& Educação, 20(4), 853-869.

Toulmin, Stephen. (2003). Regreso a la razón. Barcelona: Ediciones la península, S.A.

UNESCO. (2013). Tercer Estudio Regional Comparativo y Explicativo (TERCE). Análisis Curricular. Santiago de Chile: Laboratorio Latinoamericano de Evaluación de la Calidad de la Educación. Recuperado de http://unesdoc.unesco.org/images/0022/002275/227501s.pdf

UNESCO. (2010). Segundo Estudio Regional Comparativo y Explicativo (SERCE). Aportes para la Enseñanza de las Ciencias Naturales. Santiago de Chile: Laboratorio Latinoamericano de Evaluación de la Calidad de la Educación. Recuperado de http://unesdoc.unesco.org/images/0018/001802/180275s.pdf

UNESCO. (2015). Tercer Estudio Regional Comparativo y Explicativo (TERCE). Logros de aprendizaje. Paris, Francia. Recuperado de http://unesdoc.unesco.org/images/0024/002435/243532S.pdf

Valdez, José. (2005). Las redes semánticas naturales, uso y aplicaciones en psicología social. México: Universidad Autónoma del Estado de México. 
Vildósola, Ximena, García, Paloma y Catelló, Josep. (2005). La formación inicial del profesorado de ciencias de Chile ¿promueve la alfabetización científica en los futuros profesores? Enseñanza de las ciencias, (Extra. VII congreso), 1-7.

Vygotski, Lev. (2009). El desarrollo de los procesos psicológicos superiores. Barcelona: Biblioteca de bolsillo.

Zahur, Rubina, Barton, Angela y Upadhyay, Braskar (2002). Science education for empowerment and social change: a case study of a teacher educator in urban Pakistan. Int. J. Sci. Educ. 24(9), 899-917. 